\title{
A COMPUTATIONAL APPROACH TO PREDICT WARP OF SAWN LUMBER DUE TO RESIDUAL GROWTH STRESS IN A LOG
}

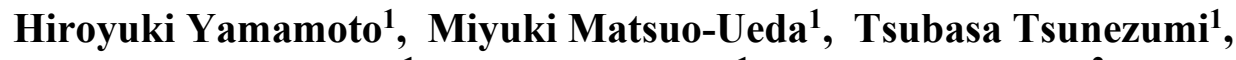 \\ Zhuoting Jiang ${ }^{1}$, Masato Yoshida ${ }^{1}$, Kana Yamashita ${ }^{2}$, \\ Yukari Matsumura ${ }^{2}$, Yosuke Matsuda ${ }^{2}$, Yuji Ikami ${ }^{2}$ \\ ${ }^{1}$ Graduate School of Bio-agricultural Sciences \\ Nagoya University \\ 464-8601, Chikusa, Nagoya, Japan \\ hiro@agr.nagoya-u.ac.jp \\ https://www.agr.nagoya-u.ac.jp/english/academics/dept1.html \\ 2 Department of Wood Properties and Processing \\ Forestry and Forest Products Research Institute \\ 305-8687, Tsukuba, Ibaraki, Japan \\ zaikana@ffpri.affrc.go.jp \\ https://www.ffpri.affrc.go.jp/en/organization/index.html
}

Key words: Sawing, Residual Stress, Growth Stress, Lumber Warp, Numerical Integration.

\begin{abstract}
A tree generates a complicated stress distribution inside the stem during its formation, which is called "residual growth stress (RGS)". The RGS often induces warp in a sawn lumber such as bow, crook, and other deformation, which causes severe losses of materials and benefits in the sawmill industry. Generally, warp becomes more serious in the lumber "without pith" than in the lumber "with pith". In Japan, 50\% of conifer plantations are ready to produce large-diameter logs of which diameter is more than $30 \mathrm{~cm}$. The use of those logs inevitably leads to the production of the lumber "without the pith", so we are concerned about the problems due to lumber warp in future sawmill industry using largediameter logs. Based on those practical background, this study presents a computational procedure to predict warp of the squared lumber, such as a beam, a bearer, and so forth, when those lumbers are sawn from the log and/or the thick plank. Using the derived procedure, 2-D patterns of the RGSs in the sawn lumbers and their changes during sawing processes are simulated. Simulated results will be experimentally verified by measuring the warp of the sawn lumber, as well as by measuring the RGS distributions in a log.
\end{abstract}




\section{INTRODUCTION}

Tree generates a 2-D stress distribution in a thin layer of the newly formed wood beneath the bark during the cell wall maturation. This is called "surface growth stress" or "growth stress". The surface growth stress induces a distribution of counteractive stress in the older wood, which is superimposed on the pre-existing stress. As a result of repetition of the thickening growth, 3-D stress distribution is induced in the log, which is called "residual growth stress (RGS)" [1,2]. A balance of the RGS in a green log is disturbed by sawing, which causes bow, crook, twist and/or other warps in the sawn lumbers. Warp of the sawn lumber is not serious when the pith is centered on the cross-section of it, as in a lumber-withpith. However, in a lumber-without-pith, the warp becomes more severe [3,4].

Many researchers consider that the warps of sawn lumber is primalily affected by the normal component of the RGS along the longitudinal direction because of a strong degree of longitudinal anisotropy in wood, and they have explained the effect of the longitudinal RGS on the warps of the primary sawing products using the elasto-mechanical and/or the finite element models [5-9]. However, the roles of the magnitude and shape of the RGS on the warps of the sawn lumbers have not been fully revealed. In addition, research has not progressed to link those theoretical/mathematical considerations to proposals for practical sawing procedures.

From that background, this study presents a computational procedure to predict warp of the long squared lumber, when those lumbers are sawn from a four-sided cant. Then,

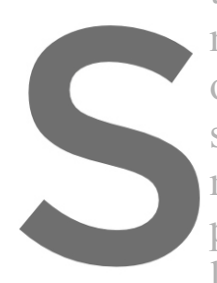
numerical experiments on the warp of the saw sawing patterns would manufacture the products front the fow. percent of the conifer plantations in Japan hav logs produced have large diameters of more than $30 \mathrm{~cm}$.

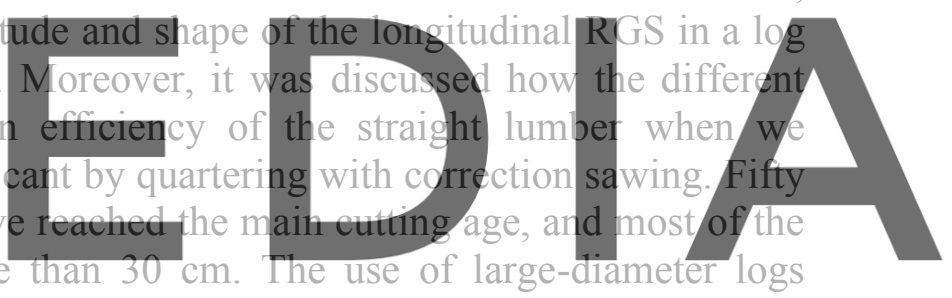
inevitably involves the production of the lumber without pith. This study is expected to

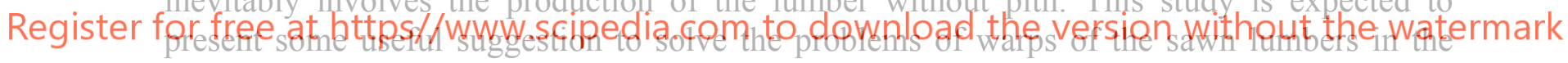
future sawmill industry.

\section{NUMERICAL MODELLING}

\subsection{Log and lumber models}

Logs A (diameter $\varphi=0.49[\mathrm{~m}])$ and B $(\varphi=0.34[\mathrm{~m}])$ with a length of $4[\mathrm{~m}]$ are virtually used that have straight and cylindrical shapes in the longitudinal direction and the circular shapes without eccentricity in the cross-section. From each log, a four-sided cant with a constant thickness and width is virtually produced as shown in Figure 1.

Each four-sided cant is further subdivided into small lumbers in a way as shown in Figure 2. An $\mathrm{O}-\xi \eta$ Cartesian coordinate system is set on the cross-section $A$ of the sawn lumber whose origin $\mathrm{O}$ is located at the geometrical centroid of $A$. At the same time, the $\zeta$-axis is set to pass through the origin $\mathrm{O}$, parallel to the pith line, so as to constitute an $\mathrm{O}-\xi \eta \zeta$ right-handed Cartesian coordinate system in the sawn lumber. It is assumed that kerf width has been neglected in the simulations for convenience. 

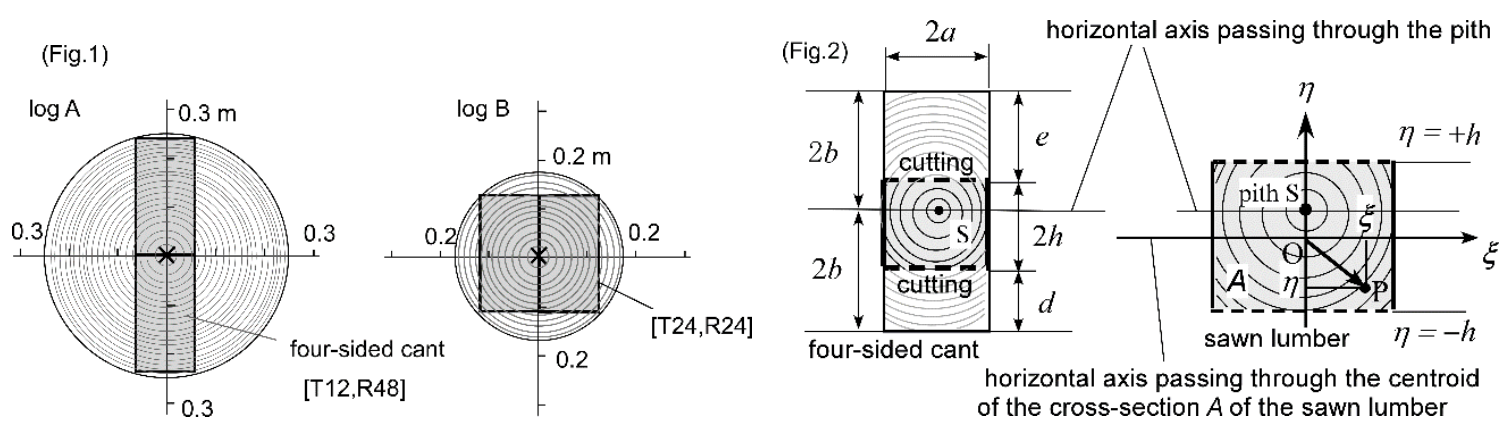

Figure 1 (left figure): Virtually introduced logs and four-sided cants. A four-sided cant having a thickness of $12[\mathrm{~cm}]$ and a width of 48 [cm] is produced from the $\log A$, and is represented as [T12, R48]. From the $\log$ B, a four-sided cant with a thickness of 24 [cm] on each side is represented as [T24, R24].

Figure 2 (right figure): A sawn squared lumber taken from the four-sided cant and $\mathrm{O}-\xi \eta$ Cartesian

coordinate system set on the domain $A$ corresponding to the cross-section of the sawn lumber. Origin $\mathrm{O}$ of the coordinate system is located at the centroid of $A$.

\subsection{Distribution of inherent strain in a $\log$}

A tree generates a distribution of the normal strain called the inherent strain $\left(\alpha_{\zeta}\right)$ on an arbitrary cross-section in the log. This induces the RGS inside the log. At an any point in the $\log , \operatorname{RGS}\left(\sigma_{\zeta}\right)$ is calculated by using the formula $\sigma_{\zeta}=-E_{\zeta} \alpha_{\zeta}$, where $E_{\zeta}$ is the Young's modulus

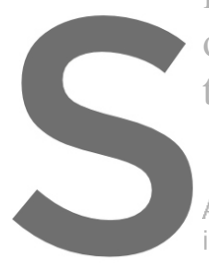
of the wood along the direction of $\zeta$-axis at that point. Many
the inherent strain can be observed as the elastic released strait
$\begin{aligned} & \text { A squared lumber is sawn at an arbitrary position } \\ & \text { in the four-sided cant. }\end{aligned}$ tesearchers have con
of the RGS [1].

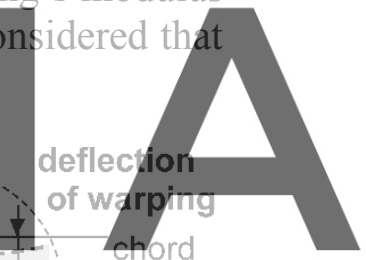

Register for free at https//www.scipedia.com to download the version without the watermark centroid of the cross-
section of the sawn lumber / pith line
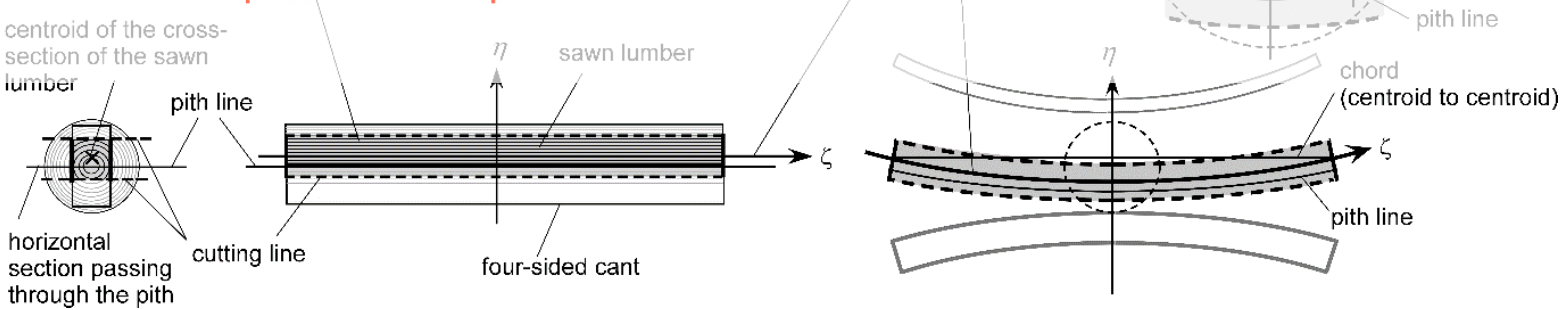

Figure 3: Measurement of deflection of the sawn lumber cut from the four-sided cant. The sawn lumber shows warp, which is measured as deflection $\delta$ at half the length of the sawn lumber.

Wood in the log shows polar anisotropy in its mechanical properties, and then both $\alpha_{\zeta}$ and $E_{\zeta}$ are distributed axisymmetrically from the pith to the bark, and uniformly along the direction of $\zeta$-axis. Therefore, the RGS $\left(\sigma_{\zeta}\right)$ will be distributed axisymmetrically in the radial direction, while uniformly along the direction of $\zeta$-axis in the log.

Sawing disturbs the balance of the RGS in a log or a lumber, which often causes lumber warps. In a four-sided cant, the remaining RGS still maintains line-symmetry with respect to both $\xi$-and $\eta$-axes on the cross-section because the origin $\mathrm{O}$ of the $\mathrm{O}-\xi \eta$ Cartesian coordinate 
system is located at the pith (see Figure 1). Therefore, no warp would occur in the four-sided cant. On the other hand, sawn lumber often shows warp in the $\eta-\zeta$ plane due to losing the linesymmetry of the remaining RGS with respect to the $\xi$-axis (see Figures 2 and 3 ). The deflection of the warp of the sawn lumber is represented by $\delta$ as shown in Figure 3, which can be simulated based on the "beam theory" as follows [10].

\subsection{How to formulate the warp of the sawn lumber cut from the four-sided cant}

The discussion is started from the following simplified constitutive equation correlating the normal stress $\sigma_{\zeta}$, the normal strain due to displacement $\varepsilon_{\zeta}$, and the inherent strain $\alpha_{\zeta}$ at an arbitrary point on the cross-section of the log, or the four-sided cant, or the sawn lumber,

$$
\sigma_{\zeta}=E_{\zeta}\left(\varepsilon_{\zeta}-\alpha_{\zeta}\right)
$$

where $E_{\zeta}$ is the Young's modulus. The strain $\varepsilon_{\zeta}$ is given as follows:

$$
\varepsilon_{\zeta}=\left\{\begin{array}{l}
0 \text { (in the log) } \\
s_{0} \quad \text { (in the four-sided cant) } \\
s_{0}+s_{1}+\varepsilon_{b} \text { (in the sawn lumber). }
\end{array}\right.
$$

where $s_{0}$ is a normal strain uniformly induced over the cross-section of the four-sided cant when it is cut from the log: $s_{1}$ is a normal strain uniformly induced over the cross-section of the sawn lumber when it is cut from the four-sided cant: $\varepsilon_{b}$ is a bending strain on the crosssection of the sawn lum

\section{Bending strain} signed distance $(\eta)$ fro 2); then, Eq.(1) can be
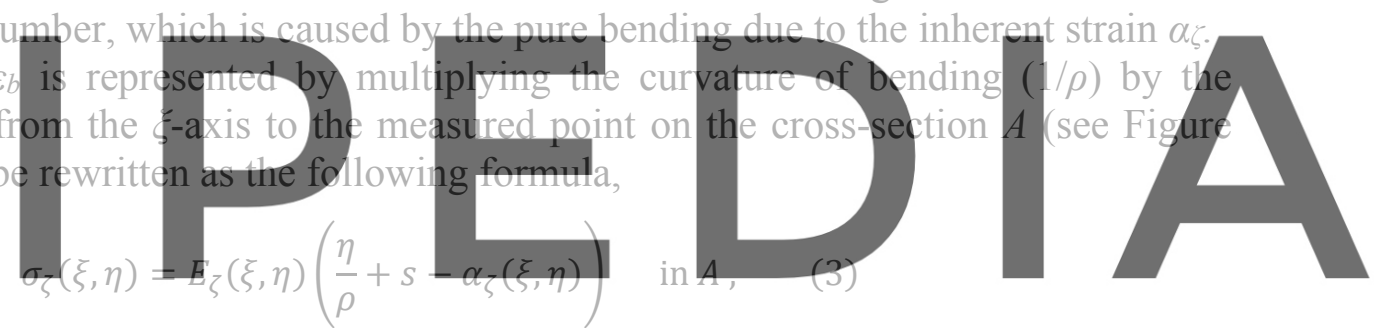

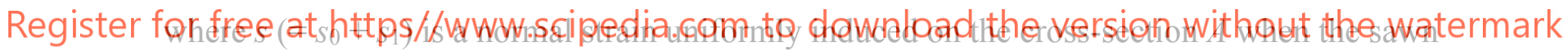
lumber is separated from "the log".

On the cross-section $A$, the axial force $N_{\zeta}$ and the bending moment $M_{\xi}$ are calculated as

$$
N_{\zeta}=\iint_{A} \sigma_{\zeta}(\xi, \eta) d \xi d \eta \quad(4), \quad M_{\xi}=\iint_{A} \sigma_{\zeta}(\xi, \eta) \eta d \xi d \eta
$$

Since the tree $\log$ is a polar anisotropic material, the neutral axis on the cross-section $A$ of the sawn lumber often deviates from the centroid. In the present study, the origin $\mathrm{O}$ of the $\mathrm{O}-$ $\xi \eta$ coordinate system is located not at the center of the neutral axis, but at the centroid of the cross-section $A$ as shown in Figure 2. The condition $N_{\zeta}=0$ is adopted as the equilibrium condition of the axial load. Furthermore, $M_{\xi}=0$ is also assumed as the equilibrium condition of the moment. Then, Eqs. (3), (4), and (5) are transformed into a system of simultaneous linear equations with respect to curvature $1 / \rho$ and axial strain $s$, which is solved as follows.

$$
\frac{1}{\rho}=\frac{I K-J H}{I^{2}-J L}, s=\frac{H I-K L}{I^{2}-J L},
$$




$$
\begin{aligned}
\text { where } I & =\iint_{A} E_{\zeta}(\xi, \eta) \eta d \xi d \eta, \quad J=\iint_{A} E_{\zeta}(\xi, \eta) d \xi d \eta, \quad K=\iint_{A} E_{\zeta}(\xi, \eta) \alpha_{\zeta}(\xi, \eta) d \xi d \eta, \\
L & =\iint_{A} E_{\zeta}(\xi, \eta) \eta^{2} d \xi d \eta, \text { and } H=\iint_{A} E_{\zeta}(\xi, \eta) \alpha_{\zeta}(\xi, \eta) \eta d \xi d \eta
\end{aligned}
$$

It is assumed that $\alpha_{\zeta}(\xi, \eta)$ and $E_{\zeta}(\xi, \eta)$ are given on the domain $A$ in advance.

\subsection{Conditions that distributions of $\alpha_{\zeta}(\xi, \eta)$ and $E_{\zeta}(\xi, \eta)$ must satisfy}

Based on the assumption of polar anisotropy, $\alpha_{\zeta}$ and $E_{\zeta}$ can be expressed as $\alpha_{\zeta}(r)$ and $E_{\zeta}(r)$, respectively, on the cross-section $A$ of the $\log$ where $\mathrm{O}-r \theta z$ cylindrical coordinate system is set. These functions must satisfy Eq.(8), which is an equilibrium condition of axial stress $\sigma_{\zeta}(r)$ on the cross-section $A$ of the log. Therefore, under the condition of Eq.(8), various $r$ dependencies are tested for $\alpha_{\zeta}(r)$ and $E_{\zeta}(r)$.

$$
\int_{A} \sigma_{\zeta}(r) d A=\int_{A}\left[-E_{\zeta}(r) \alpha_{\zeta}(r)\right] d A=-2 \pi \int_{0}^{R} E_{\zeta}(r) \alpha_{\zeta}(r) r d r=0 \quad \text { (in a log) }
$$

\section{$2.5 r$-dependencies of $\alpha_{\zeta}(r)$ and $E_{\zeta}(r)$}

With reference to the previous authors' results using sugi, $r$-dependency of $E_{\zeta}(r)$ is virtually assumed as the following conditions [11-13]. Those are (1) Es(r) is a monotonously increasing function of shape of $E_{\zeta}(r)$ depend simulation, the followin

Here, it is assumed $E_{0}=E$
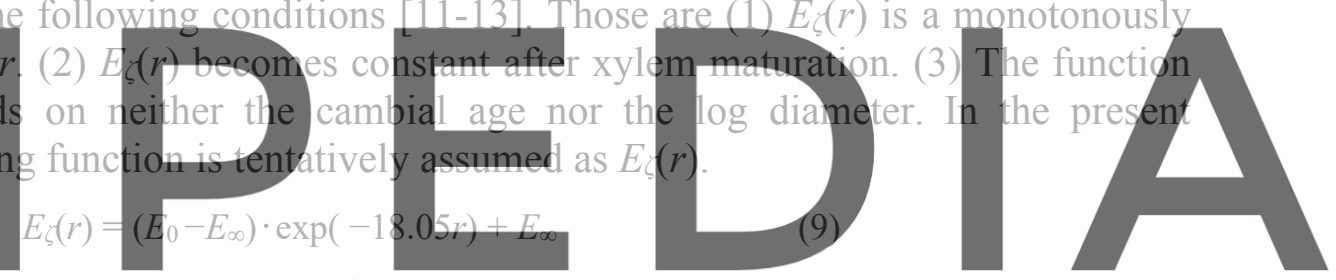

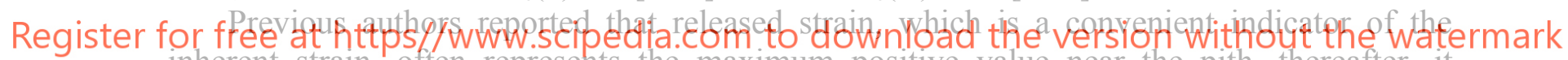
inherent strain, often represents the maximum positive value near the pith, thereafter, it monotonously decreases with the distance from the pith, and shows the minimum value (i.e., maximum negative value) at the outermost surface of the xylem (e.g., $[14,15])$. In the present study, it is assumed that the function $\alpha_{\zeta}(r)$ is monotonously decreasing for $r$. As for the magnitude of range of $\alpha_{\zeta}(r)$ in the domain of $0 \leq r \leq R$, a quantity $\Delta \alpha\left(=\alpha_{\zeta}(0)-\alpha_{\zeta}(R)\right)$ is introduced, where $\alpha_{\zeta}(0)$ is the value of inherent strain at the pith $(r=0)$, and $\alpha_{\zeta}(R)$ at the xylem surface $(r=R)$. Thus, the function forms of $\alpha_{c}(r)$ are arbitrarily assumed as follows.

(a) linear type; $\alpha_{\zeta}(r)=-\frac{\Delta \alpha}{R} r+\alpha_{\zeta}(0)$

(c) trumpet pavillon type; $\alpha_{\zeta}(r)=\frac{\Delta \alpha}{R^{2}}(r-R)^{2}+\alpha_{\zeta}(R)$

(b) Japanese bell type; $\alpha_{\zeta}(r)=-\frac{\Delta \alpha}{R^{2}} r^{2}+\alpha_{\zeta}(0)$,

In the simulation, we first assume the values of $\Delta \alpha$ and $R$ as well as the function $E_{\zeta}(r)$; thereafter, we determine the values of $\alpha_{\zeta}(0)$ and $\alpha_{\zeta}(R)$ so that $\alpha_{\zeta}(r)$ in Eq.(10) satisfies Eq.(8). Thus, the functional shape of the mechanically possible $\alpha_{\zeta}(r)$ can be determined. Figure 4 shows graphs of $\alpha_{\zeta}(r)$ in Eq.(10) to be used for the present simulation. The numbers 1 to 5 in each figure represent various values of $\Delta \alpha$, which increase in the order of $1,2, \ldots, 5$. 


\subsection{Numerical method for calculating Eq.(7)}

As shown in Figure 5, the rectangular cross-section $A$ of the sawn lumber is subdivided into $2 \times n$ vertical and $2 \times m$ horizontal, for a total of $4 \times m \times n$ microelements. The Cartesian coordinate system $\mathrm{O}-\xi \eta$ is set on the rectangular cross-section $A$. The origin $\mathrm{O}$ is the centroid of the cross-section $A$. Then, three types of coordinates are given to the point at the centroid of each microelement: "element coordinates $(i, j)$ ", "Cartesian coordinates $\left(\xi_{i}, \eta_{j}\right)$ ", and " $R$ coordinates $\left(r_{i j}\right)$ ". Suffices " $i$ " and " $j$ " take the following integers, " $-m,-m+1, \cdots,-1,0,1, \cdots$, $m-1, m$ " and " $-n,-n+1, \cdots,-1,0,1, \cdots, n-1, n$ ", respectively. The $R$ coordinate $\left(r_{i j}\right)$ means the actual distance from the pith $\mathrm{S}$ to the centroid of the $(i, j)$-th element.

(Figure 4)

(Figure 5)
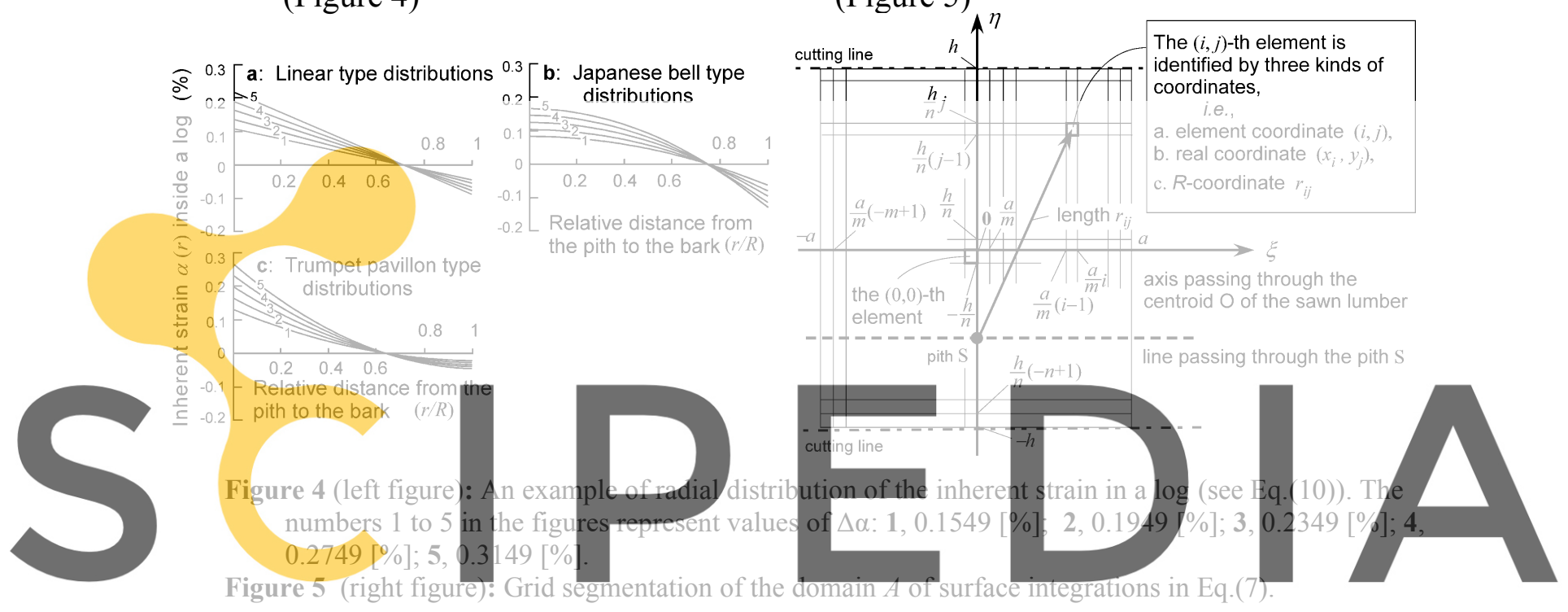

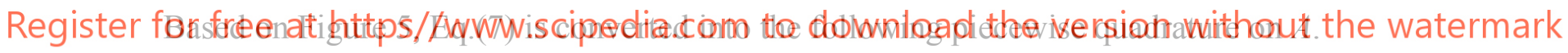

$$
I \simeq \sum_{i j} E_{i j} \eta_{j} \Delta \xi \Delta \eta, J \simeq \sum_{i j} E_{i j} \Delta \xi \Delta \eta, K \simeq \sum_{i j} E_{i j} \alpha_{i j} \Delta \xi \Delta \eta, L \simeq \sum_{i j} E_{i j} \eta_{j}{ }^{2} \Delta \xi \Delta \eta, H \simeq \sum_{i j} E_{i j} \alpha_{i j} \eta_{j} \Delta \xi \Delta \eta
$$

where $E_{i j}=E_{\zeta}\left(\xi_{i}, \eta_{j}\right), \alpha_{i j}=\alpha_{\zeta}\left(\xi_{i}, \eta_{j}\right), \Delta \xi=a / m$, and $\Delta \eta=h / n$.

When we calculate eqs.(6) using eqs.(11), the number of subdivisions $(2 m \times 2 n)$ on the cross-section $A$ must be determined in advance. When the simulation was performed assuming a plausible value for the parameter in Eq. (11), it was found that the number of subdivisions hardly affects the values of $1 / \rho$ and $s$ if $2 m \times 2 n \geq 12 \times 20(=240)$ [10].

\section{EXAMPLES OF SIMULATION}

\subsection{Examples}

Simulation 1: Changes in 2-D distributions of the RGS during the sawing process

Four-sided cants [T12, R48] and [T24, R24] were taken from the log A and B, respectively. Thereafter, each four-sided cant was cut into two equal parts (two "halved cants") along the pith (see Figures 6 and 7). In Simulation 1, 2-D distributions of the RGS in 
the cross-section of each four-sided cant before and after separation from the log, and after cutting along the pith were calculated using Eqs.(6) and (11). Results were compared among the types of the inherent strain distributions in logs.

Simulation 2: Determining the optimal sawing pattern

A four-sided cant [T12, R48] was evenly quartered followed by correction sawing as shown in Figure 8. Simulation 2 consists of two sawing patterns A and B, and each sawing pattern consists of two sawing steps. At each step, it was promised that the warp of the resulting sawn lumber must be straightened by the correction sawing before being sent to the next step or shipping if the absolute value of the deflection $(\delta)$ exceeds $8[\mathrm{~mm}]$. The criterion " $\delta_{c}=8[\mathrm{~mm}]$ " is $0.2[\%]$ of the whole length of the sawn lumber $(4[\mathrm{~m}])$, which is introduced with reference to "Japan Agricultural Standard for the structural lumber 2007". Finally, widths of the final products (= straightened quartered cants) and production yield from the four-sided cant [T12, R48] were calculated in each sawing pattern. Results were compared between two sawing patterns, as well as among the types of inherent strain distributions.

\subsection{Result of Simulation 1}

In the present calculations, an experimentally feasible value of $0.1949 \%$ was applied to $\Delta \alpha$. The results of simulations were visualized using a color bar as shown in Figures 6 and 7. In the unprocessed $\log (\boldsymbol{\alpha})$, the sign of RGS changed from compression to tension at the radial position where the $r / R$ is about $2 / 3$. In a four-sided cant which was separated from the $\log (\boldsymbol{\beta})$,

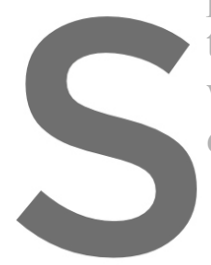

the transition point where $r / R$ is about $1 /$ direction due to separati

(a)
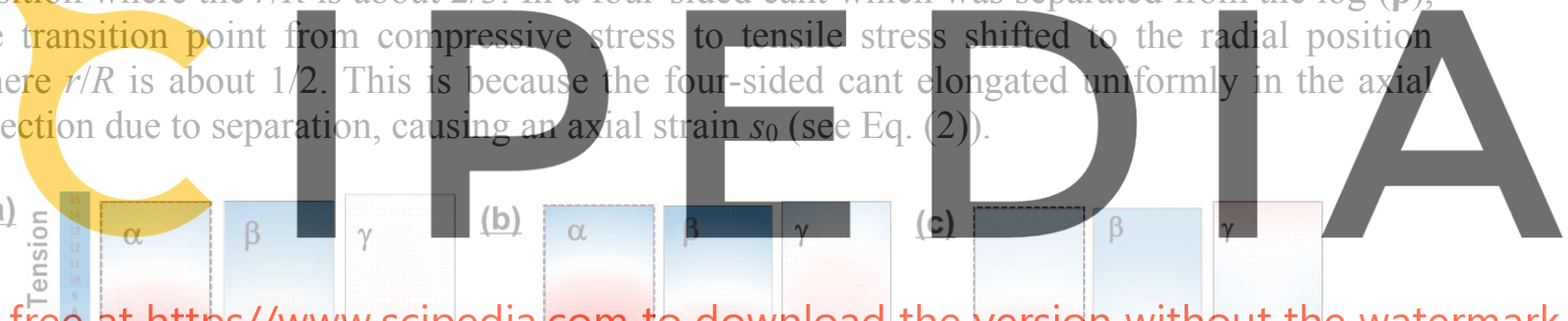

Register for free at https//www.scipedia.com to download the version without the watermark
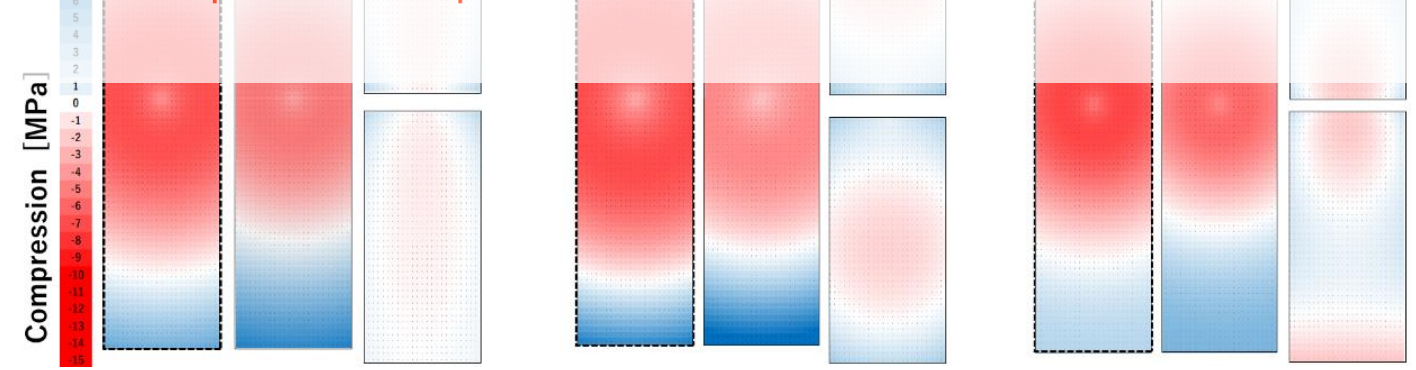

Figure 6: Result of Simulation 1. Four-sided cant [T12, R48] is cut along its pith. As the value of $\Delta \alpha$, An experimentally feasible value of 0.1949 [\%] was commonly applied in the simulation. Types of inherent strain distributions are (a) Linear, (b) Japanese bell, and (c) Trumpet pavillon. Sawing steps are $\boldsymbol{\alpha}$ : in the unprocessed $\log , \boldsymbol{\beta}$ : in the separated four-sided cant, and $\gamma$ : in the halved cant.

By cutting the four-sided cant along the pith, the resulting sawn lumber (halved cant $\gamma$ ) showed warp. At the same time, the RGS on the cross section of the halved cant was becoming more uniform. However, the state of the remaining RGS in the halved cant was 
dependent on the type of inherent strain distribution and even more greatly on the size and/or shape of the four-sided cant.

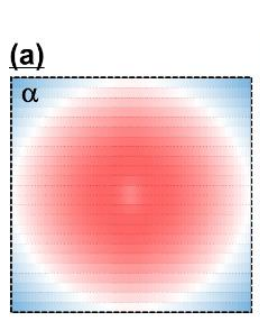

(c)

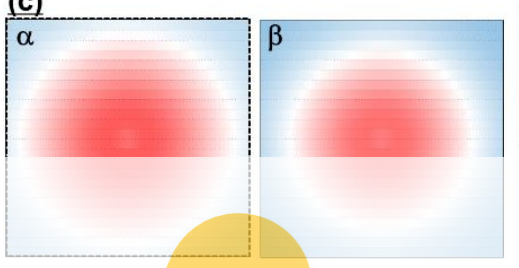

Compression $[\mathrm{MPa}]$
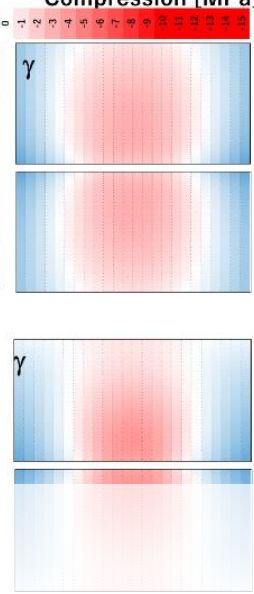

(b)

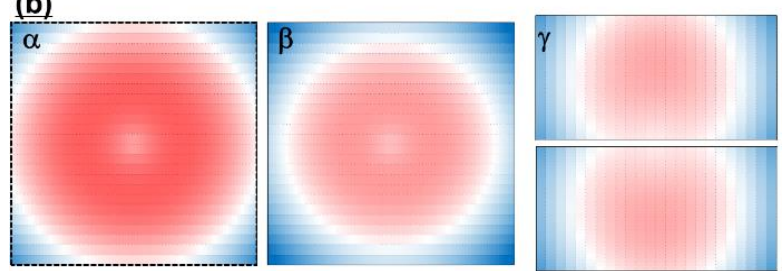

Figure 7: Result of Simulation 1. Four-sided cant [T24, R24] is cut along its pith. As the value of $\Delta \alpha$, $0.1949[\%]$ is commonly applied in the simulation. Types of inherent strain distributions are (a) Linear, (b) Japanese bell, and (c) Trumpet pavillon. Sawing steps are $\alpha$ : in the unprocessed $\log , \boldsymbol{\beta}$ : in the separated four-sided cant, and $\gamma$ : in the halved cant.

If the inherent strain distribution was linear or Japanese-bell type, weak compressive stress remained in a red-colored circular or elliptical domain centered on the centroid of the halved cant. On the other hand, if the inherent strain distribution was trumpet-pavillon type, the distribution of the remaining RGS showed rather a complicated shape. In such a case,

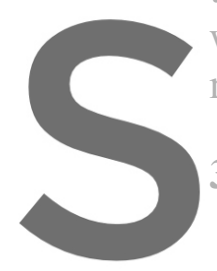
when the halved cant is further se remaining RGS.

3.3 Result of Simulation 2

In Simulation 2, an experimentally feasible
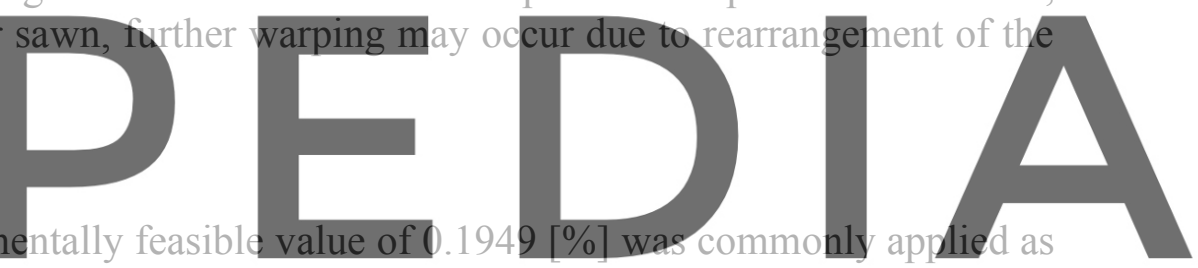

$\Delta \alpha$. At the first step of the sawing pattern A, a large part of RGS was released as shown in $\gamma$

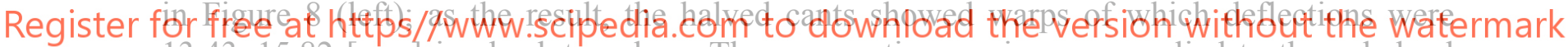

13.43 15.82 [mm] in absolute values. Thus, correction sawing was applied to those halved cants. At the second step of the sawing pattern $\mathrm{A}$, the corrected halved cant was further cut into two parts along the central axis ( $\delta$ in Figure 8 (left)). Then, warps occurred in those resulting quartered cants, of which deflections were below the criterion " $\delta_{c}=8[\mathrm{~mm}]$ " regardless of the type of the inherent strain distribution [10]. This is because more than 90 [\%] of strain energy had been already released at the first sawing step, which was noticeable in the halved cant with the inherent strain distribution of the linear type [10]. That said, deflections were marginally smaller than the criterion " $\delta_{c}=8[\mathrm{~mm}]$ " in the quartered cants with the inherent strain distributions of Japanese-bell and trumpet-pavillon types. In any case, no further correction was required at the second step in the sawing pattern A.

At the first step of the sawing pattern B, a quartered cant was taken from each bark-side of the four-sided cant, which showed warp ( $\gamma$ in Figure 8 (right)). The deflections of warps exceeded the criterion " $\delta_{c}=8[\mathrm{~mm}]$ " in the quartered cants with the inherent strain distributions of the linear and the Japanese-bell types. Thus, correction sawing was applied to those quartered cants. While, in the quartered cant with the inherent strain distribution of the trumpet-pavillon type, the deflection was marginally below than the criterion " $\delta_{c}=8[\mathrm{~mm}] "$, meaning no correction sawing is required. 
In the inner-side lumber, a large amount of RGS still remained after the first step of the sawing pattern B (see $\gamma$ in Figure 8 (right)). At the second step, it was further cut along the pith, which caused warps ( $\boldsymbol{\delta}$ in Figure 8 (right)). The deflections of those quartered cants $(=$ pith-side cants) exceeded the criterion " $\delta_{c}=8[\mathrm{~mm}]$ " in the cases with the inherent strain distributions of the linear and the trumpet-pavillon types. Correction sawing was applied to those quartered cants. While, in the case with the inherent strain distribution of the Japanesebell type, the deflection was marginally smaller than the criterion " $\delta_{c}=8[\mathrm{~mm}]$ ".

Results of Simulation 2 were summarized in Table 1. It is concluded that the Sawing pattern A is better performance than the Sawing pattern B in that it requires fewer correction sawings and that it produces lumbers as straight as possible in higher yields.

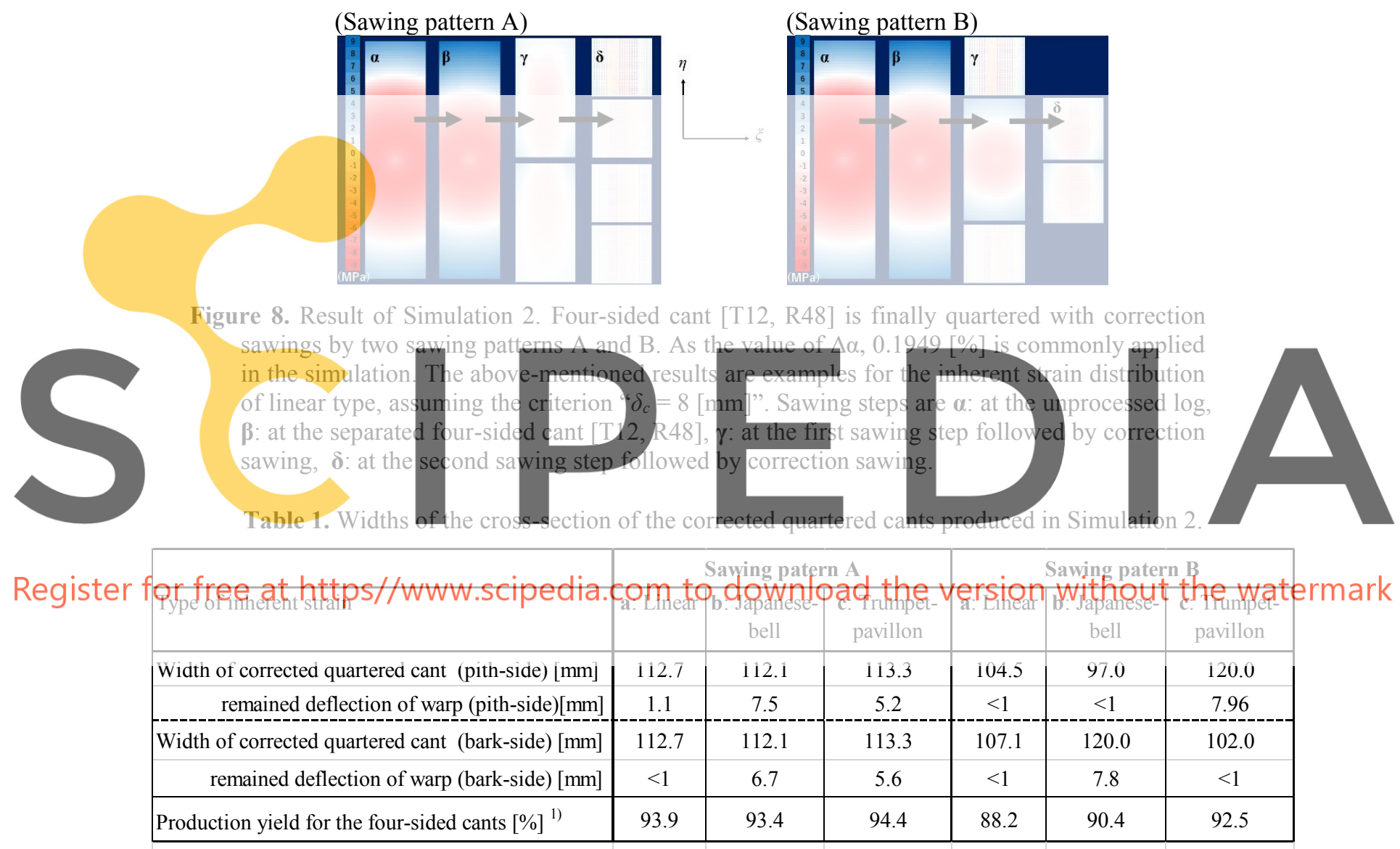

Note: 1) Volumetric ratio of production of corrected quartered cants to the four-sided cant.

\section{EXAMPLES OF VERIFICATIONS}

\subsection{Examples}

Experiment 1: Warp of sawn lumber when the four-sided cant was cut along the pith

Fifteen green logs of sugi (Cryptomeria japonica) with a top diameter of $0.37 \sim 0.42[\mathrm{~m}]$ and a length of $4[\mathrm{~m}]$, planted in Ibaraki, Japan, were used. Each log were devided into two logs with a length of 2 [m]. From the top half log, a four-sided cant with thickness of 12 [cm] 
was sampled, which was further cut into two halved cants along the pith. Then, deflection of warp due to cutting $(\delta)$ was measured at half the length of each halved cant by using a dialgage device, which was transformed into the curvature $1 / \rho$ by the formula,

$$
\frac{1}{\rho}=\frac{8 \delta}{L^{2}+4 \delta^{2}} \approx \frac{8 \delta}{L^{2}}, \quad \delta \approx \frac{L^{2}}{8}\left(\frac{1}{\rho}\right),
$$

where $L(=1.5[\mathrm{~m}])$ is the span length of the device.

From the bottom half log, a quartersawn board with thickness of 4 [cm] was taken, and normal component of the released strain in $\zeta$ direction $\left(\hat{\alpha}_{\zeta}\right)$ was measured by the strain gauge method. Measurement points were set radially every $2 \mathrm{~cm}$ from the pith to both bark sides $[14,15]$. Thereafter, a rectangular specimen with a size of $10(\xi$-width $) \times 5$ ( $\eta$-thickness $) \times 100$ $\left[\mathrm{mm}^{3}\right]$ ( $\zeta$-length) was taken from each measurement point of the released strain, and was used for measurement of the Young's modulus in $\zeta$ direction $\left(\hat{E}_{\zeta}\right)$ by the tensile test.

We assumed that both $\hat{\alpha}_{\zeta}$ and $\hat{E}_{\zeta}$ are axisymmetric with respect to the pith, and that both radial distribution patterns are the same in the top and bottom logs. The value of $\hat{\alpha}_{\zeta}$ was tentatively adopted as the inherent strain $\left(\alpha_{\zeta}\right)$ in each tested log. Both $\hat{\alpha}_{\zeta}$ and $\hat{E}_{\zeta}$ were applied to Eqs. (6) and (11) to simulate the curvature of warp of the halved cant.

Experiment 2: Four-sided cants were evenly quartered in three sawing patterns

In this experiment, total 33 four-sided cants were prepared from 33 green logs of sugi (Cryptomeria japonica) with a top diameter of $0.34 \sim 0.38[\mathrm{~m}]$ and a length of $2[\mathrm{~m}]$, which

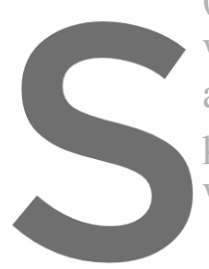
were evenly devided into three groups. As show
assigned to test the three types of sawing patte
pattern, the warp due to cutting was measure
with an asterisk after cutting in the order of the (Experiment 1)
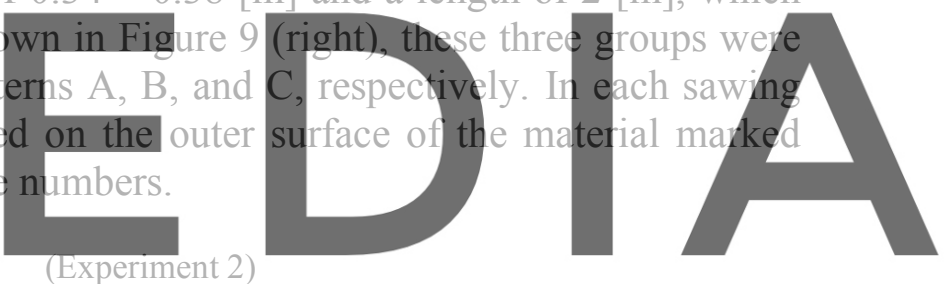

Register for log with a length of 4 [m] Sawn pattern A pattern B
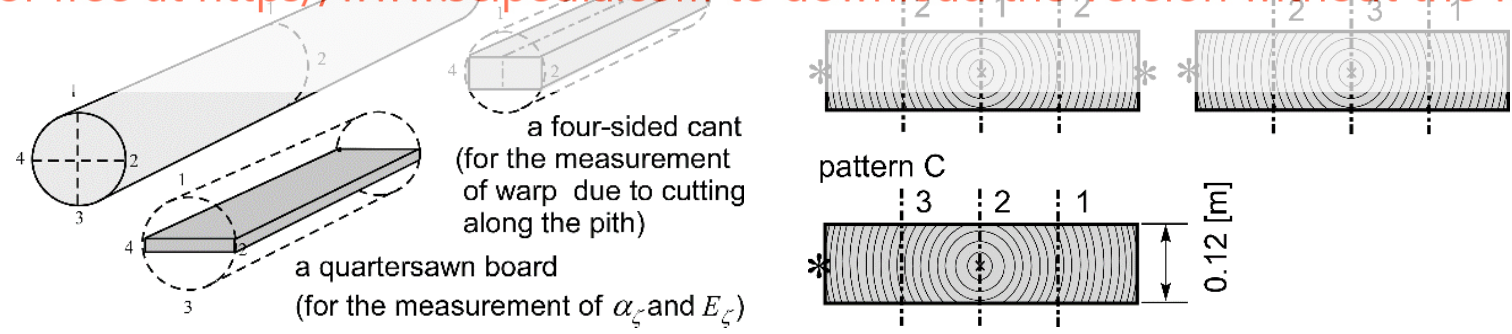
pattern C

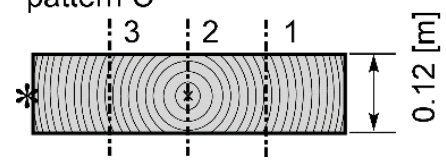

Figure 9: Scheme of experiment. In Experiment 1 (left figure), we examined whether the observed curvatute of the halved cant could be reproduced by applying the measured values of $\hat{\alpha}_{\zeta}$ and $\hat{E}_{\zeta}$ to Eqs.(6) and (11). In Experiment 2 (right figure), the warp is measured on the outer surface of the material marked with an asterisk just after cutting in the order of the numbers.

\subsection{Result of Experiment 1}

As can be seen from Figure 10, experimental results of the curvatures of the halved cants were highly corelated with the simulated results: this means that the warp of sawn lumber can be reproduced from the measured distributions of the inherent strain and the Young's modulus by the simulation using Eqs. (6) and (11). At the same time, Figure 10 gives a certain 
propriety to the assumption that $\hat{\alpha}_{\zeta}$ and $\hat{E}_{\zeta}$ have axisymmetric distributions in a tested $\log$, which has been supported by the authors' separate experiment even with some errors and exceptions [16].

The simulation gave more or less lower values in the curvature of warp in the halved cant than the observation.

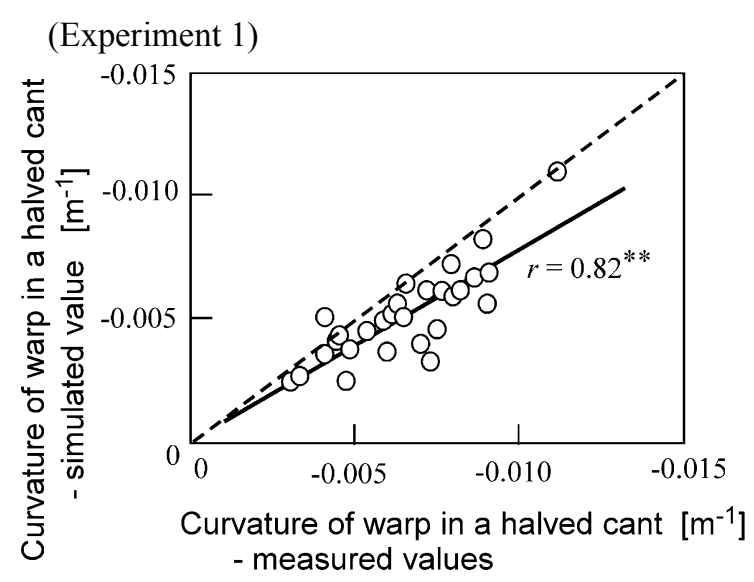

Figure 10: Results of verifications by Experiment 1 (left figure) and Experiment 2 (right figure) using large-diameter logs of sugi (Cryptomeria japonica).

\subsection{Result of Experiment 2}

As can be seen from Figure 10 (right), the deflection of warp depended on the sawing pattern. In Sawing pattern A, a large warp was generated by the cutting at the first sawing step (" 1 " in the uppermost figure), and as a result, the RGS was almost released (see Figure 8). The average warp newly generated at the second sawing step (" 2 " in the uppermost figure) was almost 0 ; however, if anything, it can be said that the warp that has occurred at often recovered slightly at this stage.

In the sawing patterns $\mathrm{B}$ and $\mathrm{C}$, a certain amount of the RGS still remained at the first sawing step because the sawn lumber was not yet divided along the pith. The remaining RGS will be released at the subsequent step, and the additional warp also increased. Considering that the warp must be corrected by correction sawing, in the Sawing pattern A, if correction sawing is performed immediately after " 1 ", it is no more required at the subsequent step " 2 ". Considering the number of operation of the correction sawing, it can be said that the Sawing pattern A was the most optimal among three tested sawing patterns.

Acknowledgement: Present paper consists of a part of published reference [10] and authors' unpublished results [16]. The research was supported by grants from the Project of the Bio-oriented Technology Research Advancement Institution, NARO (the special scheme project on advanced research and development for next-generation technology).

\section{REFERENCES}

[1] Archer, R.R. Growth stresses and strains in trees. Springer-Verlag, (1986). 
[2] Kübler, H. Growth stresses in trees and related wood properties. For. Prod. Abstr. (1987) 10: $62-119$.

[3] Ikami, Y.; Murata, K.; Matsumura Y.; Tsuchikawa, S. Influence of pith location on warp of lumber in sawing medium-qualitty sugi (Cryptomeria japonica D. Don) logs. Eur. J. Wood Prod. (2009) 67: 271-276.

[4] Matsumura, Y.; Murata, K.; Ikami, Y.; Matsumura, J. Influence of sawing patterns on lumber quality and yield in large sugi (Cryptomeria japonica D. Don) logs. For. Prod. J. (2012) 62: 25-31.

[5] Okuyama, T. and Sasaki, Y. Crooking during lumbering due to residual stresses in the tree. Mokuzai Gakkaishi (1979) 25: 681-687.

[6] Vendhan, C.P. and Archer, R.R. Relief of growth stress in diametral planks. Holzforschung (1977) 31: 90-96.

[7] Bandyopadhyay, N. and Archer, R.R. Relief of growth stresses in planks. Holzforschung (1979) 33: 43-46.

[8] Ormarsson, S.; Dahlblom, O.; Johansson, M. Finite element study of growth stress formation in wood and related distortion of sawn lumber. Wood Sci. Technol. (2009) 43: 387-403.

[9] Johansson, M. and Ormarsson, S. Influence of growth stress and material properties on distortion of sawn timber - numerical investigation. Ann. For. Sci. (2009) 66: Article No. 604.

[10] Yamamoto, H.; Matsuo-Ueda, M.; Tsunezumi, T.; Yoshida, M.; Yamashita, K.; Matsumura, Y.; Matsuda, Y.; Ikami, Y. Effect of residual stress distribution in a log on lumber warp due to sawing. - A numerical simulation based on the beam theory. Wood Science and Technology (2021) 55: 125-153.

[11] Yamashita, K.; Hirakawa, Y.; Fujisawa, Y.; Nakada, R. Effects of microfibril angle and density on variation of modulus of elasticity of sugi (Cryptomeria japonica) logs among eighteen cultivars (in Japanese). Mokuzai Gakkaishi (2000) 46:510-522.

[12] Matsumura, Y.; Murata, K.; Ikami, Y.; Omori, M.; Matsumura, J. Application of the wood properties of large-diameter Sugi (Cryptomeria japonica) logs to sorting logs and sawing patterns. J. Wood Sci. (2013) 59: 271-281.

[13] Ando, K.; Mizutani, M.; Toba, K.; Yamamoto, H. Dependence of Poisson's ratio and Young's modulus on microfibril angle (MFA) in wood. Holzforschung (2017) 72: 321327.

[14] Tejada, A.; Okuyama, T.; Yamamoto, H.; Yoshida, M. Reduction of growth stress in logs by direct heat treatment. Assessment of a commercial-scale operation. For. Prod. J. (1997) 47: 88-93.

[15] Wahyudi, I.; Okuyama, T.; Hadi, Y.S.; Yamamoto, H.; Yoshida, M.; Watanabe, H. Growth stress and strain of Acacia mangium. For. Prod. J. (1999) 49: 77-81.

[16] Authors' unpublished results. 